\title{
EVALUATING WORKLOAD ASSOCIATED WITH TELEMATIC DEVICES VIA A SECONDARY TASK PROTOCOL
}

\author{
Mustapha Mouloua, Edward Rinalducci, Peter A Hancock. J Christopher Brill \\ University of Central Florida \\ Psychology Dept., P.O. Box 161390 \\ 4000 Central Florida Blvd. \\ Orlando, FL 32816-1390 \\ E-mail: mouloua@pegasus.cc.ucf.edu
}

\begin{abstract}
There are a variety of driver distractions that negatively affect driver workload and performance. These distractions range attempting to light a cigarette, and putting on make-up, to eating or drinking, tuning the radio, using a cellular phone, or using an in-vehicle navigation system. Of particular present interest are the distracting effects of telematic devices, which include traffic information systems, telecommunication, intelligent aid and control, and navigational systems. These devices can now be found on-board various types of U.S. and foreign automobiles. Despite having many potential benefits, there are also several behavioral problems resulting from poor use of these devices. The present research was designed to investigate the deleterious effects of telematics on driver performance. It was hypothesized that all the telematic systems used in this study would degrade driver performance and increase workload.
\end{abstract}

A mixed-model factorial design ( $2 \times 3)$ was used, with telematics being a between-subject factor and allocation phase a within-subject factor (repeated measures). All participants were required to drive three, four-minute simulated (pre, during, and post) allocation phases. In the preallocation phase, participants were required to drive while performing a secondary counting task, (counting and responding to a series of randomly presented visual signals). During the allocation phase, participants were required to drive and perform the secondary counting task while either talking on the phone or tuning a radio (distractibility task). In the post-allocation phase, participants were required to drive while performing the secondary counting task. Data from the counting task (number of correct, wrong, and misses) and driving errors (collisions, crossing the median, leaving the road, maintaining the speed limit, and lane deviations) were recorded and statistically analyzed.

Thirty-four participants (nine males and 25 females) from the University of Central Florida participated in this study. A series of analyses of variance (ANOVA) were conducted to test for the effects of telematics and workload on each of the dependent measures. A significant main effect of phase on lane deviations was observed, $F(2,64)=10.58, p<.001$, indicating that more lane deviations were made during the cell phone and radio tuning use $(M=9.14)$ than during both of the pre-allocation $(M=4.14)$ and post-allocation $(M=5.88)$ phases. ANOVA also yielded a significant main effect of phase on crossing the median, $F(3,68)=4.63, p<.05$, indicating that more crossings were made during the allocation phase $(M=5.05)$ than during the pre-allocation $(M=3.05)$ and post-allocation $(M=4.47)$ phases. Similarly, the results also showed a significant effect of phase on the distraction task performance, $F(2,64)=5.70, p<.01$, indicating that more errors were made during the allocation phase $(M=6.50)$ than during the pre-allocation $(M=4.50)$ and the post-allocation $(M=3.38)$ phases. 
The present findings indicate that both cellular phone and radio systems are capacity demanding. The counting task results demonstrate the increased level of workload associated with these telematic devices. In addition, driving performance errors were also higher for both the cellular phone and the radio systems. Our findings suggest the need to regulate the use of such devices in order to avoid overloading the driver's attentional spare capacity. 